\title{
GCU
}

Glasgow Caledonian

University

University for the Common Good

\section{Erasmus Mundus intercultural competence - results and EMIC Toolkit}

Lukic, D.; Solari, M.; Martins, H.; Yarosh, M.

Published in:

EDULEARN17 Proceedings

DOI:

10.21125/edulearn.2017.1897

Publication date:

2017

Document Version

Author accepted manuscript

Link to publication in ResearchOnline

Citation for published version (Harvard):

Lukic, D, Solari, M, Martins, H \& Yarosh, M 2017, Erasmus Mundus intercultural competence - results and EMIC Toolkit. in EDULEARN17 Proceedings. IATED, pp. 4177-4183, EDULEARN17, Barcelona, Spain, 3/07/17. https://doi.org/10.21125/edulearn.2017.1897

\section{General rights}

Copyright and moral rights for the publications made accessible in the public portal are retained by the authors and/or other copyright owners and it is a condition of accessing publications that users recognise and abide by the legal requirements associated with these rights.

Take down policy

If you believe that this document breaches copyright please view our takedown policy at https://edshare.gcu.ac.uk/id/eprint/5179 for details of how to contact us. 


\title{
ERASMUS MUNDUS INTERCULTURAL COMPETENCE - RESULTS AND EMIC TOOLKIT
}

\author{
D. Lukic ${ }^{1}$, M. Solari ${ }^{2}$, H. Martins ${ }^{3}$, M. Yarosh ${ }^{4}$ \\ ${ }^{1}$ Glasgow Caledonian University - GCU London (UNITED KINGDOM) \\ ${ }^{2}$ University of Poitiers (FRANCE) \\ ${ }^{3}$ University of Porto (PORTUGAL) \\ ${ }^{4}$ University of Deusto (SPAIN)
}

\begin{abstract}
The paper reports on the final results and outputs of the Erasmus Mundus Intercultural Competence project (EMIC). The EMIC project was designed to develop an integrated learning programme in order to help Erasmus Mundus students deal with issues of cultural diversity and, from a developmental perspective, use intercultural contexts to their advantage. The learning intervention was contextualised within students' own experience, to allow them to compare the theoretical concepts with situations encountered in everyday study and life. The learning intervention was evaluated by means of a mixed methods approach. Qualitative and quantitative data were collected, in order to improve the EMIC Learning Programme and develop a practical and feasible Toolkit output for developing Intercultural Competence (IC).

The paper details main features of the EMIC Toolkit which was the final output of the project. Based on the EMIC project experience, the EMIC Toolkit is proposed as an Open Education Resource (OER) that is scalable, customisable and potentially useful for other higher education programmes. The purpose of the EMIC Toolkit is to provide a set of flexible resources and tools to support developing IC in contexts similar to the Erasmus Mundus programme. The Toolkit is composed of a series of learning activities organised in 'bundles' so that they can be used as a reflection tool to support students in developing IC. Activities are arranged in a blended learning model with a proposed mix of face-to-face workshops and individual and group reflective online activities. The EMIC Toolkit also offers easy-to-use strategies for customising and implementing contextualised learning activities in the users' own cultural and academic contexts through guidelines and examples of good practices. The paper concludes with notes on implementation further pathways to develop the IC approaches in higher educational institutions.
\end{abstract}

Keywords: Intercultural Competence, toolkit, blended learning, joint degrees, higher education.

\section{INTRODUCTION}

The mobility that the Erasmus Mundus joint Master Courses (EMMCs) provides is one of its most important characteristics. Students gain educational experience in at least two countries that have different teaching and learning models. Students also get to live in culturally diverse settings while moving through two or more countries during their Erasmus Mundus studies. Having to both study and live in several different contexts can present intercultural requiring a set of abilities for dealing with such diversity [1]. These abilities, collectively termed 'Intercultural Competence' (IC) [2], enable effective studying, living and working across different contexts. Only situating people from different countries together in a culturally diverse context does not automatically help develop IC. Moreover, there is a danger of individuals developing negative responses to cultural misunderstandings and experiences if they are not prepared.

Therefore, proper support and training might be needed to fully utilise the potential for innovative learning that the cultural diversity of a study abroad experience can provide. The Erasmus Mundus IC (EMIC) project was designed to develop an integrated learning programme in order to help Erasmus Mundus students deal with issues of cultural diversity and, from a developmental perspective, use intercultural contexts to their advantage. Learning is contextualised within students' own experience, to allow them to compare the theoretical concepts with situations encountered in everyday study and life.

Based on the EMIC project experience, this Toolkit is proposed as an Open Education Resource (OER) that is scalable, customisable and potentially useful for other higher education programmes. 
The EMIC Toolkit provides a set of resources and tools to support developing IC in contexts similar to the Erasmus Mundus programme. Despite the fact that the EMIC Toolkit was developed based on experiences of joint Master's students from around the world studying in European Higher Educational Institutions, the approach tested and described in the Toolkit addresses core IC that is relevant for all students experiencing mobility internationally or home students studying in diverse settings, as well as in preparation for intercultural workplace experiences in their careers.

\section{METHODOLOGY}

The EMIC Toolkit was developed within the Erasmus Mundus IC (EMIC) Project funded by the European Commission under the Erasmus Mundus Action 3 fund. The project included several phases: Baseline Study, Learning Intervention, Evaluation, EMIC Toolkit Development including the Dissemination phase throughout the project. The paper present the final results and outputs of the project that were informed by data collected in all phases of the project:

1. The Baseline study was a qualitative phase based on 42 stakeholders' interviews.

2. The Intervention Phase was a blended learning programmer tested with 31 student participants from four Erasmus Mundus Master students

3. The Evaluation phase used a mixed method approach collecting questionnaires pre- and postintervention and collecting interviews with participants and trainers in the project

4. The EMIC Toolkit Development phase was informed by the results of all the 3 previous phases and further collected feedback form the wider Steering Group, including participating EMMCs consortia.

Four EMMCs were involved in this project: GIM (Global Innovation Management), MALLL (Master of Arts in Lifelong Learning), EUROMIME (European Master in Media Engineering for Education) and WOP-P (Work, Organisations and Personnel Psychology). Students were invited to participate either directly through email or via contact points from the different EMMCs; 31 students enrolled distribution of participants regarding their EMMCs is shown in Figure 1.

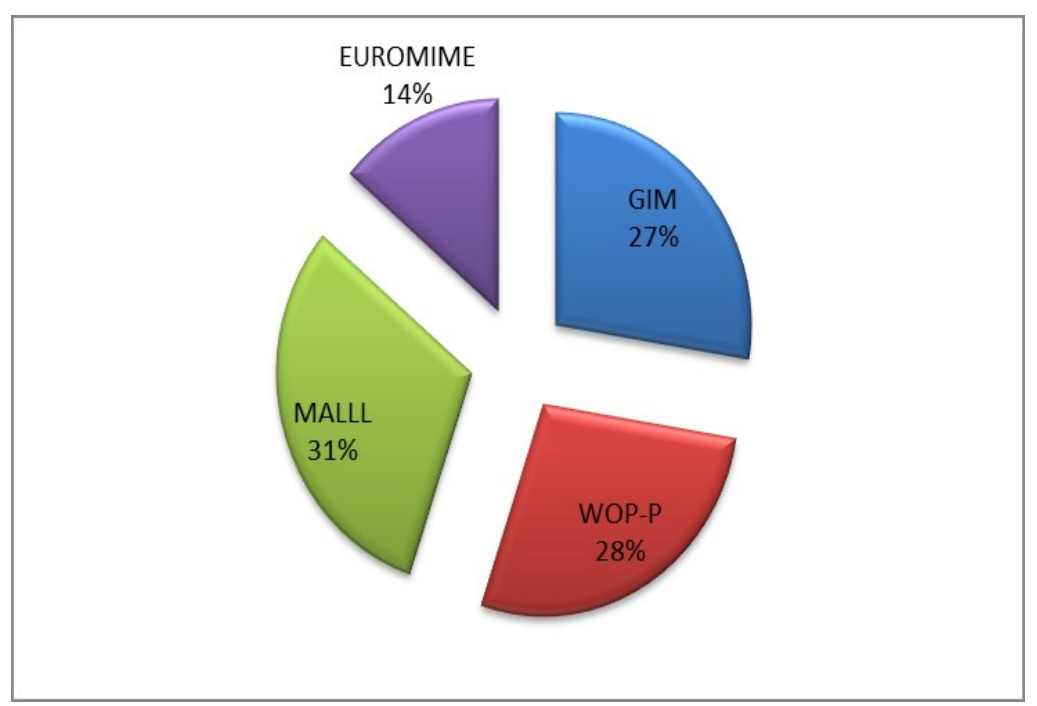

Figure 1 - Participants of the learning programme according to their course

\section{RESULTS}

Based on the training recommendations, particular learning needs in terms of content, literature review and research owns training experience, the EMIC Learning Programme was developed within the project. The EMIC Learning Programme is aimed at further developing student' IC for a competitive international labour market and mobility. The Learning Programme is based on EMIC Learning Outcomes developed within the project. The EMIC Learning Programme consists of:

- 2 day face-to-face Workshop 
- Engagement with the EMIC Online Learning Platform (interactive learning activities, learning resources - estimated from 8 to 10 hours of students workload)

- Filling an EMIC questionnaire before and after the training to evaluate impact of the Learning programme

The EMIC Learning Programme tested in the Intervention Phase uses an interactive blended learning approach that focuses on reflection and use of student experience as the basis for personal and professional development $[3,4]$. The methodology focuses on a training customised for student needs to help them improve their IC. The training was delivered by team approved by the European Commission within the EMIC Project. The training team had a range of experiences in competence development in academic and workplace settings.

\subsection{Intervention phase impact}

The EMIC Learning Programme aimed at helping students in EMMC's further develop their ICs through a training with a face to face and an online modality. Our evaluation indicated that the participants were highly satisfied with training and that the training does seem to have an impact on their competence.

Regarding the face to face workshop, students highlighted the fun and interactive activities and the preparedness of trainers and mentioned they would have preferred to receive more information prior to the training concerning the theoretical models. Training timing seemed to be quite important and variable; this might mean that other trainers might want to negotiate the best timing with the students and academics involved in the Masters they engage.

The online portion of the training experienced a degree of dropout. Students that concluded the training referenced it as a positive experience that allowed for more in depth reflection than the face to face workshop, but a lack of interaction with others and collaborative learning. All students that concluded the online training had had previous experience with this modality of training. Students suggested the sending of weekly reminders of the training, which we believe might be a good practice for others replicating this training.

Most participants provided a more beneficial review on the impact of the face-to-face portion of the learning programme than on the online activities. The feedback from the group was that inclusion of two cohorts from the same Master programmes was an advantage and added that, in the future, students from different cohorts or even programmes would bring added value to the course. Students felt that the topic of IC development benefited from the online platform, despite finding the engagement with this portion of the training harder than the face-to-face part. Some found the face-toface training helped them more with the emotional component of IC, whereas the online part was useful with the more cognitive part of the training [5]. All interviewed students mentioned the training was useful for them on a personal level. The study indicated that the EMIC learning programme has the potential to be a very useful support for anyone aiming at developing students' IC, and should be adapted to the specificities of the training context [6].

\subsection{EMIC Toolkit}

The project resulted in a number of relevant outputs (EMIC Theoretical Approach, Baseline Study Concise Report, EMIC Learning Programme, EMIC Learning Platform, EMIC Round Table Presentation and EMIC Evaluation Report - all available at http://www.emic-project.org/outcomes). However, the main project output that is informed by all other results and outputs is the EMIC Toolkit which is an Open Education Resource (OER) that is scalable, customisable and potentially useful for all European higher education programmes (full Toolkit available at http://www.emic-project.org/emictoolkit). The purpose of the EMIC Toolkit is to provide a set of flexible resources and tools to support developing IC in Erasmus Mundus and similar international student mobility programmes.

The EMIC Toolkit also offers easy to use strategies for customising and implementing contextualised learning activities in the users' own cultural and academic contexts through guidelines and examples of good practices.

\subsubsection{Who is the Toolkit for}

The EMIC Toolkit is based on the experience of joint Master students (Erasmus Mundus or otherwise) and is therefore primarily beneficial for: 
- Higher Educational Institutions who want to develop support programmes for developing IC of their joint Master students;

- Departments in charge of student experience for Master students (international departments, support units, academic development centres and similar)

- Teachers and Lecturers who deal with international Master students and want to integrate IC development in their teaching [7];

- Individual Master students who want to develop their IC and/or organise similar activities with their colleagues.

Although the EMIC Toolkit was developed based on experiences of joint Master students from around the world studying in European Higher Educational Institutions, the learning programme described in this Toolkit addresses core IC that is relevant for all students experiencing mobility internationally or home students studying in diverse settings, as well as preparation for intercultural workplace experiences in their careers. Therefore, the EMIC Toolkit could be useful and adapted for:

- Higher Educational Institutions who want to develop support programmes for their students in general (whether international students, home students in diverse classroom setting or for preparation for career in intercultural workplaces; undergraduate, postgraduate or even $\mathrm{PhD}$ students);

- Departments in charge of student experience at all levels;

- Higher education Teachers and Lecturers who deal with diverse educational contexts;

- Individual students who want to develop their IC and/or organise similar activities with their colleagues for better academic experience and employability.

Although the EMIC Toolkit has been designed to facilitate the EMIC Learning Programme adaptation, it is important to consider that some challenges may arise when used in settings different from those where it was created. The EMIC Learning Programme has only been used with study-abroad students in joint master programmes; it is important to consider this before using it for study-abroad students with only one host country or with interethnic groups belonging to one unique country.

\subsubsection{Benefits of using the EMIC Toolkit}

- Experience an interdisciplinary and research based learning;

- Get an opportunity to reflect on personal development needs and development plans in terms of IC;

- Gain knowledge, skills, awareness and attitudes needed for high level IC;

- Develop specific skills such as intercultural conflict management, emotional intelligence and intercultural relationship building needed both for academic and professional work;

- Get an opportunity to share experiences and learn from other participants;

- Improve academic experience in diverse contexts;

- Increase employability as IC is one of top competences all employers look for, especially in international settings [8].

Our project showed that the Toolkit has a great deal of potential for institutions and individuals and some organisations are already freely using the OER resources.

\subsubsection{Added value of the EMIC Toolkit}

There are a variety of tools and resources for skills and knowledge related to interculturality. In contrast to other programmes that develop IC, the EMIC Toolkit is based on a unique approach to learning development that is:

- interdisciplinary, integrating current knowledge on formal learning in higher education, nonformal learning in the non-profit sector, workplace learning and technology-enhanced learning;

- based on scientific research, from previous studies on intercultural learning and from data gathered throughout the EMIC Project; 
- customised, that is, based on specific training needs of joint Master students;

- contextualised in participants experiences, using the experiences as the 'content' of learning;

- flexible, that is, learning activities are designed as modifiable and applicable bundles across a range of different contexts and cultural situations; and

- open-source, allowing other Erasmus Mundus programs to implement and/or modify the proposed EMIC Learning Programme in their courses.

\subsubsection{Facilitators guidelines}

The Toolkit contains two types of guidelines for offering an effective IC learning programme: implementation of guidelines for institutions or individuals that aim to organise the learning programme within their context, and facilitator's guidelines for those specifically involved in leading activities with participants that can aid all relevant stake-holders in utilising the main output of the EMIC project. In order to create the conditions for the EMIC Learning Programme implementation, facilitators are recommended to consider the following aspects:

Ambiance: The atmosphere should be relaxed, friendly and cooperative, while the programme itself should be perceived as fun, rather than more workload. To create a fun, friendly and relaxed ambiance it is important to maintain a horizontal facilitators-participants relationship and to keep reminding participants that $\mathrm{IC}$ is a life-long-learning process.

Educational context: Facilitators should keep in mind different educational contexts involved (both local and those from student backgrounds). Any potential contradictions in educational cultures should be brought up and discussed with participants.

Experience as content: The diversity of the group and the richness of the participants' individual experiences must be used and built on $[9,10]$. This can be done by encouraging participants to share their experiences. Facilitators can also elicit participants' opinions on difference-rich situations, and thus bring them to everyone's attention.

Guidance rather than recipes: Facilitators should also avoid giving participants 'answers' to the questions participants have, but rather help them discover strategies for finding the answers on their own (with the help of their classmates/representatives of different cultures).

Relevance of activities: Facilitators should make sure participants see the relevance and see how to link what they learn in the learning programme to their everyday reality. One of the key aims of facilitation and feedback given by the facilitators is to make students reflect critically aware of their own IC level and development needs, and to encourage participants realizations that there is always room for improvement [11].

Perceived expertise: Facilitators should build a rapport and understand issues the students have been facing. For the same reason, facilitators must be cautious not to focus on only one particular culture because it might compromise/modify the interaction with the representatives of this culture.

Ethos: Facilitators should be aware that IC has a close link to personal identity; this is why many activities can move easily from cultural to personal level. Facilitator has to be careful not to go too deep into the private sphere. However, without going into therapy settings, facilitators should be able to create an emotionally safe zone (free of judgment, open-minded, etc.) to discuss personal issues and to ensure participants that the information will not be used against them after the learning programme.

\subsection{EMIC project lessons learned}

Through our experience in the EMIC project we developed a range of good practices, lessons learned and discoveries that might be relevant for other similar international projects. The individuals involved in the project as well as their institutions, learned a great deal from the projects successes and challenges from which all definitely emerged as better researchers and practitioners on the account of the process. However, there are several practices that could benefit the wider community:

- Releasing outcomes and outputs as an open source to enable access to everyone and therefore a wider engagement from the academic and practitioner community. 
- Making sure that project teams are interdisciplinary and intersectoral and focus on their interchange of knowledge in order to bridge the knowledge silos problem in education research and development [1].

- Use of shared cloud folders (such as Dropbox) for collaborative editing and work. This element was very important to ensure proper communication, co-creation of knowledge and document management.

- Use of real life experiences as content of educational programs, making the learning process more experiential and customised for individual learner needs $[3,4]$.

- Focusing on scientific methods, even if project funding guidelines focus more on the development side rather than the research aspect of the problem. This ensures sound knowledge and implementation as well as acceptance by the wider scientific community.

- Making sure that collaborators are trusted colleagues that can show critical thinking, challenge their colleagues a support each other in project difficulties.

Such aspects of the project enable successful completion and production of outputs that are viable long-term.

\section{CONCLUSIONS}

The EMIC Toolkit, as an adaptable and OER set of tools, has a potentially high impact on a number of HEl stakeholders. The first impact of the Toolkit was felt in four of the participating institutions, through dissemination to their international offices and departments in charge of learning and teaching development. The projects tested showed a clear impact on the participating sample. The Toolkit was disseminated wider through project networks, networks of EMMCs and Erasmus Mundus Association and a number of institutions started using the tools. However, as the EMIC Toolkit was the last output for the project and its long term impact is yet to be seen empirically, the project partnership has decided to stay in close contact with future implementers through use of the EMIC website. However, as it is an OER openly available to everyone and attribution cannot be guaranteed some impacts and uses of the Toolkit might be missed. This is an issue with OER tools generally. Despite the fact that the EMIC Toolkit was developed based on experiences of joint Master's students from around the world studying in European Higher Educational Institutions, the learning programme described in this Toolkit addresses core IC that is relevant for all students experiencing mobility internationally or home students studying in diverse settings, as well as in preparation for intercultural workplace experiences in their careers. The study also showed that IC of university academic and administrative staff might need further exploration since the pressure for developing this competence in student is increasing [8]. Further studies would show what are the benefits and challenges of adapting the EMIC Toolkit resources and activities to the profession development of educational practitioners.

\section{ACKNOWLEDGEMENTS}

The project team would like to thank the European Commission Erasmus Mundus Action 3 Unit without whose support the project would not have been possible. We would also like to thank all four participating EMMC consortia and colleagues for their collaboration on the project. We would also like to thank the Erasmus Mundus Association for their support.

\section{REFERENCES}

[1] Lukic, D., Yarosh, M., \& Martins, H., "Intercultural Competence: Bridging the Intersectoral Gap", Proceedings of the 5th International Technology, Education and Development Conference (INTED2011), Published by IATED. ISBN (CD): 978-84-614-7423-3, 2011.

[2] Fantini, A., "A Central Concern: Developing Intercultural Competence", School for International Training Occasional Papers Series, Inaugural Issue, pp. 25-42, 2000. Retrieved from http://www.adam-europe.eu/pri/2935/prd/8/1/develop-l-com.pdf

[3] Schön, D. A., The Reflective Practitioner: How Professionals Think in Action, London: Temple Smith, 1983.

[4] Glowacki-Dudka, M. and Barnett, N., "Connecting Critical Reflection and Group Development in Online Adult Education Classrooms", International Journal of Teaching and Learning in Higher Education, 19(1), pp. 43-52, 2007. 
[5] Lustig, M. W. and Koester, J., Intercultural Competence: Interpersonal Communication Across Cultures, Boston, New York, etc.: Allyn \& Bacon, 2003.

[6] Lukic, D., Solari, M. \& Yarosh, M., "Developing Intercultural Competence in Erasmus Mundus: Reflective Practice Through Technology-enhanced Learning", Proceedings of the EDULEARN2015 Conference, Barcelona, Spain: IATED, 2015.

[7] Lukic, D., Yarosh, M., Solari, M. \& Martins, H., "Intercultural Competence of University Employees in Erasmus Mundus Joint Degrees", Proceedings from the 9th International Conference on Researching Work and Learning (RWL), Singapore, Paper 045. (2015). Retrieved from http://www.rwl2015.com/pdf/RWL9-Conference-Booklet.pdf

[8] Gregersen-Hermans, J. "Intercultural Competence Development in Higher Education" in Intercultural Competence in Higher Education, International Approaches, Assessment and Applications (Deardorff, D. \& Asarathanam, L. eds.), Routledge, 2017.

[9] Deardorff, D. K., "A 21st Century Imperative: Integrating Intercultural Competence in Tuning", Tuning Journal for Higher Education, 3(1), pp. 137-147, 2015.

[10] Fantini, A., Exploring and Assessing Intercultural Competence. Research report. Washington, D. C.: Center for social development, Global service institute, 2007.

[11] Verpoorten, D., Westera, W. and Specht, M., "Using Reflection Triggers While Learning in an Online Course", British Journal of Educational Technology, 43, pp.1030-1040, 2012. 